\title{
Inertial effects in the orientational relaxation of rodlike molecules in a uniaxial potential
}

\author{
Yuri P. Kalmykov, ${ }^{1, a)}$ Serguey V. Titov, ${ }^{2}$ and William T. Coffey ${ }^{3}$ \\ ${ }^{1}$ Laboratoire de Mathématiques, Physique et Systèmes, Université de Perpignan, 52, Avenue de Paul Alduy, \\ 66860 Perpignan Cedex, France \\ ${ }^{2}$ Kotel'nikov Institute of Radioengineering and Electronics, Russian Academy of Sciences, \\ Vvedenskii Square 1, Fryazino 141190, Russian Federation \\ ${ }^{3}$ Department of Electronic and Electrical Engineering, Trinity College, Dublin 2, Ireland
}

(Received 18 November 2008; accepted 5 January 2009; published online 13 February 2009)

\begin{abstract}
The inertial rotational Brownian motion and dielectric relaxation of an assembly of noninteracting rodlike polar molecules in a uniaxial potential are studied. The infinite hierarchy of differential-recurrence relations for the equilibrium correlation functions is generated by averaging the governing inertial Langevin equation over its realizations in phase space. The solution of this hierarchy for the one-sided Fourier transforms of the relevant correlation functions is obtained using matrix continued fractions yielding the longitudinal dipole correlation function, the correlation time, and the complex polarizability, which are calculated for typical values of the model parameters. Pronounced inertial effects appear in these characteristics in the high-frequency region for low damping. The exact longitudinal correlation time is compared with the predictions of the Kramers theory of the escape rate of a Brownian particle from a potential well as extended by Mel'nikov and Meshkov [J. Chem. Phys. 85, 1018 (1986)]. In the low temperature limit, the universal Mel'nikov and Meshkov formula for the inverse of the escape rate provides a good estimate of the longitudinal correlation time for all values of the dissipation including the very low damping, very high damping, and Kramers turnover regimes. Moreover, the low-frequency part of the spectra of the longitudinal correlation function may be approximated by a single Lorentzian with a halfwidth determined by this universal escape rate formula. (C) 2009 American Institute of Physics.
\end{abstract}

[DOI: 10.1063/1.3074330]

\section{INTRODUCTION}

The rotational Brownian motion of rodlike molecules in a double-well uniaxial (Meier-Saupe) potential

$$
V(\vartheta)=-\sigma k_{B} T \cos ^{2} \vartheta
$$

where $\sigma$ is the dimensionless anisotropy parameter, $k_{B}$ is Boltzmann's constant, $T$ is the temperature, and $\vartheta$ is the polar angle, is almost invariably used to describe molecular reorientations in nematic liquid crystals (see, e.g., Refs. 1-8) and biological membranes (e.g., Refs. 9 and 10). However, the vast majority of treatments have been confined to the noninertial or very high damping (VHD) limit due to the mathematical difficulties associated with the rotation of a rigid body in space. The dynamics of the system in this limit have been extensively studied by using the associated Smoluchowski equation (see, e.g., Refs. 2-6 and references cited therein). In dielectric relaxation, the inertial effects of molecules are unimportant at low frequencies. However, neglect of the molecular inertia leads to incorrect predictions of the behavior of dielectric spectra at high frequencies (e.g., infinite integral absorption). ${ }^{8}$ Existing treatments accounting for inertial effects have been given either by numerical simulation $^{11,12}$ or under the rather unrealistic assumption of rotation about a fixed axis. ${ }^{13,14}$ We remark that mathemati-

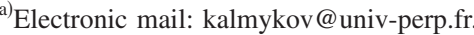

cally speaking the dielectric response of polar molecules in a mean field potential closely resembles magnetic relaxation of uniaxial single domain ferromagnetic particles, $8,15,16$ and the dynamic Kerr effect of nonpolar polarizable molecules. ${ }^{8,17}$

Now, the theoretical treatment of inertial rotational Brownian motion has been usually based on the associated Fokker-Planck equation. ${ }^{18-20}$ That equation, in the present context, is a partial differential equation for the time evolution of the joint distribution function of orientations and angular momenta of a molecule in phase space. It is derived by calculating the drift and the diffusion coefficients from the inertial Euler-Langevin equation which governs the time behavior of the set of random variables describing the rotational Brownian motion of a molecule in a fluid. The solution of the Fokker-Planck equation is usually obtained by separating the variables. A comprehensive discussion of that equation and its applications to orientational relaxation in fluids is given, e.g., in Refs. 18-20. However, an alternative approach has been given by Coffey $^{21}$ and Coffey et al. ${ }^{8}$ They developed a method of solution of the Langevin equations for the inertial Brownian rotation without recourse to the Fokker-Planck equation. The key step in applying the method is first to convert by appropriate transformation the Langevin equation into an equation for the quantity the statistical average of which is desired, i.e., the observable. That equation is then averaged over its realizations yielding differential-recurrence relations for the time behavior of the 
statistical averages from which the equilibrium correlation functions may be deduced. Now, the transformed Langevin equation contains not only the desired average but also the next higher order average and so on. It is thus the generating equation of a hierarchy of averages which can be solved by a variety of methods. This procedure entirely eliminates the excessive step in the theory of constructing and solving the corresponding Fokker-Planck equation. The advantage in computational labor that the direct averaging method based on the Langevin equation has over the solution by the Fokker-Planck method is considerable because neither the derivation of that equation nor a knowledge of the intricate transformations used to effect separation of the variables in it is required. These advantages have been highlighted in a recent paper $^{22}$ in the context of the three-dimensional rotational Brownian motion in a potential.

Here we shall use a recently developed method ${ }^{22}$ for the evaluation of the inertial response of systems of polar molecules in a double-well uniaxial potential, Eq. (1), with dynamics described by the inertial Langevin equation, which is based on an exact solution of the associated recurrence relations for the spectra of the statistical moments using matrix continued fractions and which also allows one to derive approximate analytic solutions by utilizing the concepts of the integral relaxation (correlation) time, escape rate, and the effective eigenvalue. In particular, we shall present a detailed comparison of Mel'nikov and Meshkov's universal turnover formula for the longest relaxation time with the matrix continued fraction solution for the longitudinal dipole correlation function, the correlation time, and the dynamic susceptibility.

\section{RECURRENCE RELATIONS FOR STATISTICAL AVERAGES}

We consider the three-dimensional rotational diffusion of a thin rod, or rotator, representing a typical linear polar molecule in the Meier-Saupe uniaxial anisotropy potential Eq. (1). In the molecular coordinate system oxyz fixed in the rotator, the angular velocity $\boldsymbol{\omega}=\left(\omega_{x}, \omega_{y}, \omega_{z}\right)$ and the angular momentum $\mathbf{M}=\left(I \omega_{x}, I \omega_{y}, 0\right)$ of the rotator are defined as ${ }^{1}$

$$
\boldsymbol{\omega}=(\dot{\vartheta}, \dot{\varphi} \sin \vartheta, \dot{\varphi} \cos \vartheta), \quad \mathbf{M}=(I \dot{\vartheta}, I \dot{\varphi} \sin \vartheta, 0),
$$

where $\varphi$ is the azimuthal angle. The rotational Brownian motion of the rotator is governed by the Euler-Langevin equations, ${ }^{8,18}$

$$
\begin{aligned}
& I \dot{\omega}_{x}(t)=I \omega_{y}^{2}(t) \cot \vartheta(t)-\zeta \omega_{x}(t)-\partial_{\vartheta} V[\vartheta(t)]+\lambda_{x}(t), \\
& I \dot{\omega}_{y}(t)=-I \omega_{x}(t) \omega_{y}(t) \cot \vartheta(t)-\zeta \omega_{y}(t)+\lambda_{y}(t),
\end{aligned}
$$

where $-\partial_{\vartheta} V[\vartheta(t)]$ is the torque due to the external potential $V,-\zeta \omega_{x}(t)$ and $-\zeta \omega_{y}(t)$ are the components of the frictional torque $\zeta \boldsymbol{\omega}(t)$, and $\lambda_{x}(t)$ and $\lambda_{y}(t)$ are the components of the white noise torque $\boldsymbol{\lambda}(t)$ due to the Brownian motion of the surroundings having the following properties:

$$
\overline{\lambda_{j}(t)}=0, \quad \overline{\lambda_{j}(t) \lambda_{m}\left(t^{\prime}\right)}=2 k_{B} T \zeta \delta_{j, m} \delta\left(t-t^{\prime}\right) .
$$

Here $j, m=x, y, z$, and the overbar means the statistical average over an ensemble of rotators that all start at the instant $t$ with the same sharp values of the angular velocity and the orientation.

Our goal is to evaluate the longitudinal dipole correlation function $C(t)$ defined as

$$
C(t)=\frac{\langle\cos \vartheta(0) \cos \vartheta(t)\rangle}{\left\langle\cos ^{2} \vartheta(0)\right\rangle}
$$

where the angular brackets mean the statistical average over the equilibrium distribution function $W_{0}$ of $\vartheta, \varphi, \omega_{x}$, and $\omega_{y}$,

$$
\langle(\cdot)\rangle=\int_{-\infty}^{\infty} \int_{-\infty}^{\infty} \int_{0}^{2 \pi} \int_{0}^{\pi}(\cdot) W_{0} \sin \vartheta d \vartheta d \varphi d \omega_{x} d \omega_{y} .
$$

Here $W_{0}\left(\vartheta, \omega_{x}, \omega_{y}\right)=Z^{-1} e^{-\eta^{2}\left(\omega_{x}^{2}+\omega_{y}^{2}\right)+\sigma \cos ^{2} \vartheta}$ is the MaxwellBoltzmann distribution associated with the set of rotators, $\eta=\sqrt{I /\left(2 k_{B} T\right)}, I$ is the moment of inertia of a rotator, and $Z$ is the partition function. Having determined $C(t)$ from Eq. (4), one may calculate the correlation (or integral relaxation) time $\tau$ defined as the area under the curve of the correlation function, viz.,

$$
\tau=\int_{0}^{\infty} C(t) d t
$$

and the longitudinal complex susceptibility $\chi(\omega)=\chi^{\prime}(\omega)$ $-i \chi^{\prime \prime}(\omega)$ defined as ${ }^{8}$

$$
\frac{\chi(\omega)}{\chi^{\prime}(0)}=1-i \omega \widetilde{C}(\omega)
$$

where $\chi^{\prime}(0)=\left(\mu^{2} N_{0} / k_{B} T\right)\left\langle\cos ^{2} \vartheta(0)\right\rangle$ is the static susceptibility and $N_{0}$ is the number of dipoles per unit volume.

By averaging the inertial Langevin Eqs. (2) and (3), we have derived in Ref. 22 the infinite hierarchy of differentialrecurrence relations for the correlation functions $c_{n}^{l, m}(t)$ $=\left\langle\cos \vartheta(0) f_{n}^{l, m}(t)\right\rangle$ describing the relaxation of the system in an arbitrary axially symmetric potential $V(\vartheta)$. Here

$$
f_{n}^{l, m}(t)=P_{l}^{m}[\cos \vartheta(t)] s_{n}^{m}\left[\omega_{x}(t), \omega_{y}(t)\right],
$$

$P_{l}^{k}(z)$ are the associated Legendre functions, ${ }^{23}$ the orthogonal functions $s_{n}^{m}\left(\omega_{x}, \omega_{y}\right)$ (Refs. 8 and 22) are expressed as finite series of products of Hermite polynomials $H_{n}(z)$ (Ref. 23) in the components of the angular velocity (representing the generalization by induction of a method described in Ref. 21), viz.,

$$
s_{n}^{2 m+M}\left(\omega_{x}, \omega_{y}\right)=\sum_{q=0}^{n} \frac{r_{2 m+M}^{n, q}}{q !(n-q) !} H_{2 n-2 q+M}\left(\eta \omega_{x}\right) H_{2 q}\left(\eta \omega_{y}\right),
$$

where $M=0,1$ and the coefficients $r_{2 m+M}^{n, q}$ are to be determined from the following recurrence relations:

$$
\begin{aligned}
r_{2 m}^{n, q}= & \left(n-q+\frac{1}{2}\right)\left(1-\frac{2 q+1}{2 m-1}\right) r_{2 m-1}^{n, q} \\
& +(n-q) \frac{2 q+1}{2 m-1} r_{2 m-1}^{n, q+1},
\end{aligned}
$$




$$
r_{2 m+1}^{n, q}=\left(1+\frac{q}{m}\right) r_{2 m}^{n, q}-\frac{q}{m} r_{2 m}^{n, q-1},
$$

with $r_{0}^{n, q}=r_{1}^{n, q}=1$; thus one has ${ }^{8} r_{2}^{n, q}=n-2 q, r_{3}^{n, q}=n-4 q$, $r_{4}^{n, q}=n(n-1)-8 q(n-q)$, etc. For the uniaxial anisotropy (Meier-Saupe) potential, Eq. (1), these differentialrecurrence equations become for $m=0$,

$$
\begin{aligned}
\eta \frac{d}{d t} c_{n}^{l, 0}= & -2 n \beta^{\prime} c_{n}^{l, 0}+\frac{1}{2} c_{n}^{l, 1}+\left(2+\frac{4 \sigma}{(2 l-1)(2 l+3)}\right) c_{n-1}^{l, 1} \\
& -\frac{4 \sigma}{2 l+1}\left(\frac{l}{2 l-1} c_{n-1}^{l-2,1}-\frac{l+1}{2 l+3} c_{n-1}^{l+2,1}\right),
\end{aligned}
$$

and for $m \geq 1, M=0,1$,

$$
\begin{aligned}
\eta \frac{d}{d t} c_{n}^{l, 2 m-M}= & -(2 n+M) \beta^{\prime} c_{n}^{l, 2 m-M}+c_{n-1+M}^{l, 2 m+1-M}+\frac{1}{4} c_{n+M}^{l, 2 m+1-M}-(l+2 m-M)(l-2 m+1+M) \\
& \times\left[\frac{n-m+1+M}{4} c_{n+M}^{l, 2 m-1-M}+(n+m) c_{n-1+M}^{l, 2 m-1-M}\right]+2 \sigma\left\{\frac{(l+1-2 m+M)}{(2 l+1)(2 l+3)} c_{n-1+M}^{l+2,2 m+1-M}+\frac{(4 m-2 M+1)}{(2 l-1)(2 l+3)} c_{n-1+M}^{l, 2 m+1-M}\right. \\
& -\frac{(l+2 m-M)}{(2 l-1)(2 l+1)} c_{n-1+M}^{l-2,2 m+1-M}-(n+m)\left[\frac{(l-2 m+1+M)(l-2 m+2+M)(l-2 m+3+M)}{(2 l+1)(2 l+3)} c_{n-1+M}^{l+2,2 m-1-M}\right. \\
& -\frac{(4 m-2 M-1)(l-2 m+1+M)(l+2 m-M)}{(2 l-1)(2 l+3)} c_{n-1+M}^{l, 2 m-1-M} \\
& \left.\left.-\frac{(l+2 m-2-M)(l+2 m-1-M)(l+2 m-M)}{(2 l-1)(2 l+1)} c_{n-1+M}^{l-2,2 m-1-M}\right]\right\},
\end{aligned}
$$

where $\beta^{\prime}=\zeta \eta / I$ is the dimensionless damping parameter.

Equations (7) and (8) can be solved by taking the onesided Fourier transform and then using matrix continued fractions ${ }^{8}$ (see Appendix). Having determined the one-sided Fourier transform $\widetilde{c}_{0}^{1,0}(i \omega)=\int_{0}^{\infty} c_{0}^{1,0}(t) e^{-i \omega t} d t$, we can calculate the spectrum of the correlation function $\widetilde{C}(\omega)$ as

$$
\tilde{C}(\omega)=\frac{\widetilde{c}_{0}^{1,0}(i \omega)}{c_{0}^{1,0}(0)}
$$

and the correlation time $\tau$, viz.,

$$
\tau=\widetilde{C}(0)=\frac{\widetilde{c}_{0}^{1,0}(0)}{c_{0}^{1,0}(0)} .
$$

Equations (7) and (8) can also be derived after lengthy calculations from the inertial Fokker-Planck equation for the distribution function $W\left(\vartheta, \omega_{x}, \omega_{y}, t\right)$, which is ${ }^{18}$

$$
\frac{\partial W}{\partial t}=L_{\mathrm{FP}} W,
$$

where the Fokker-Planck operator $L_{\mathrm{FP}}$ is defined as

$$
\begin{aligned}
L_{\mathrm{FP}}= & -\omega_{x} \partial_{\vartheta}-\omega_{y} \cot \vartheta\left(\omega_{y} \partial_{\omega_{x}}-\omega_{x} \partial_{\omega_{y}}\right)+I^{-1} \partial_{\vartheta} V \partial_{\omega_{x}} \\
& +\frac{\beta^{\prime}}{\eta}\left[\partial_{\omega_{x}}\left(\omega_{x}+\frac{1}{2 \eta^{2}} \partial_{\omega_{x}}\right)+\partial_{\omega_{y}}\left(\omega_{y}+\frac{1}{2 \eta^{2}} \partial_{\omega_{y}}\right)\right] .
\end{aligned}
$$

\section{ASYMPTOTIC FORMULAS FOR $\tau$ IN THE LOW TEMPERATURE LIMIT}

In the present context, which involves a symmetric double-well potential the correlation time $\tau$ is essentially the inverse of the smallest nonvanishing eigenvalue $\lambda_{1}$ of the Fokker-Planck operator Eq. (12). In other words, $\lambda_{1}^{-1}$ is the lifetime of the slowest relaxation mode of the system. However, $\lambda_{1}$ is not, in general, available in closed form because it is invariably the smallest nonvanishing root of a very high order polynomial equation, namely, the secular equation of the system. Fortunately, a way of overcoming this difficulty is to utilize an ingenious method originally proposed by Kramers $^{24}$ in connection with thermally activated escape of particles out of a potential well. Kramers ${ }^{24}$ evaluated the prefactor $\mu$ in an Arrhenius-type equation for the escape rate $\Gamma$ over the potential barrier $\Delta V$, viz.,

$$
\Gamma=\mu \Gamma_{\mathrm{TST}},
$$

where $\Gamma_{\mathrm{TST}} \sim\left(\omega_{0} / 2 \pi\right) e^{-\Delta V / k_{B} T}$ is the transition state theory (TST) escape rate and the attempt frequency $\omega_{0}$ is the angular frequency of a particle executing oscillatory motion at the bottom of a well (for reviews of applications of Kramers' method see Refs. 25-27). Now if the escape rates for mechanical Brownian particles are calculated by the Kramers method, three regimes of damping appear, viz., (i) intermediate-to-high damping (IHD) containing within it the VHD region, (ii) very low damping (VLD), and (iii) a turnover region. Kramers ${ }^{24}$ mentioned in his paper, however, that he could not find a general method of attack for the purpose of obtaining a formula which would be valid for any damping regime. This problem known as the Kramers turnover 


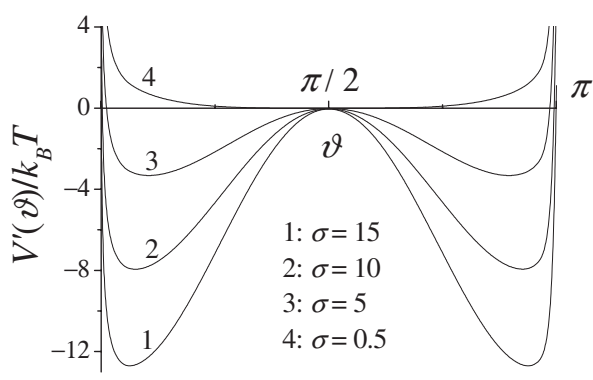

FIG. 1. Effective potential $V^{\prime}(\vartheta) / k_{B} T=-\sigma \cos ^{2} \vartheta-\ln \sin \vartheta$.

problem was resolved nearly 50 years later by Mel'nikov and Meshkov. ${ }^{28,29}$ By solving the Fokker-Planck equation converted to an energy-action diffusion equation by the WienerHopf method, they ${ }^{28,29}$ evaluated the longest relaxation time $\tau$ in the high barrier limit for all values of the dissipation, in terms of a depopulation factor (which is a function of the ratio of the energy loss per cycle of a particle moving on an escape trajectory to the thermal energy), which heuristically interpolates between the VLD and IHD regions. In other words, they effectively solved the Kramers turnover problem. Moreover, Mel'nikov ${ }^{29}$ extended the depopulation factor method to take into account quantum effects in a semiclassical way. Later, Grabert ${ }^{30}$ and Pollak et al. ${ }^{31}$ presented a comprehensive solution of the classical Kramers turnover problem, showing that the Mel'nikov and Meshkov universal formula can be obtained without their ad hoc interpolation between the weak and strong damping regimes. We remark that the calculation of Pollak et al. ${ }^{31}$ also applies to an arbitrary memory friction and not only to the "white noise" (memoryless) limit. Later their calculation was extended to the quantum regime in the semiclassical limit by Rips and Pollak. $^{32}$

The universal turnover formula of Mel'nikov and Meshkov as applied to the present problem has been obtained by Pastor and Szabo ${ }^{12}$ as follows. In order to estimate the prefactor $\mu$ in Eq. (13), they averaged the exact inertial FokkerPlanck Eq. (11) over the angular velocity component $\omega_{y}$, which allowed them to transform Eq. (11) to a simpler Fokker-Planck equation for the reduced distribution function $W^{\prime}\left(\vartheta, \omega_{x}, t\right)$, viz.,

$$
\begin{aligned}
\frac{\partial W^{\prime}}{\partial t} & +\omega_{x} \frac{\partial W^{\prime}}{\partial \vartheta}-\frac{1}{I} \frac{\partial V^{\prime}}{\partial \vartheta} \frac{\partial W^{\prime}}{\partial \omega_{x}} \\
= & \frac{\beta^{\prime}}{\eta} \frac{\partial}{\partial \omega_{x}}\left(\omega_{x} W^{\prime}+\frac{1}{2 \eta^{2}} \frac{\partial W^{\prime}}{\partial \omega_{x}}\right),
\end{aligned}
$$

where

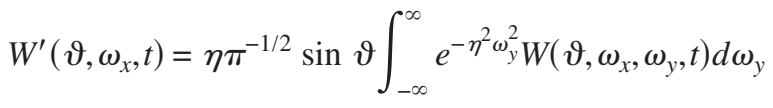

and $V^{\prime}(\vartheta)=V(\vartheta)-k_{B} T \ln \sin \vartheta$ is an effective potential (see Fig. 1). Here the potential $V^{\prime}(\vartheta)$ is infinite at 0 and $\pi$, has a maximum at $\vartheta=\pi / 2$, and so resembles a typical onedimensional bistable potential in the range $0<\vartheta<\pi$. The reduced Fokker-Planck equation Eq. (14) describes onedimensional Brownian rotation about a fixed axis in the effective potential $V^{\prime}$. Thus, the Mel'nikov-Meshkov depopu- lation factor method is directly applicable, and one obtains for the longest relaxation time $\tau_{M}{ }^{12}$

$$
\tau_{M} \sim \frac{\eta \omega_{b} A\left[2 \beta^{\prime} S\right]}{\Gamma_{\mathrm{TST}} A^{2}\left(\beta^{\prime} S\right)}\left(\sqrt{4 \eta^{2} \omega_{b}^{2}+\beta^{\prime 2}}-\beta^{\prime}\right)^{-1} .
$$

Here, $\omega_{b}=\sqrt{\left|\partial_{\vartheta}^{2} V^{\prime}(\pi / 2)\right| / I}=\eta^{-1} \sqrt{\sigma-1 / 2}$ is the barrier frequency, $S$ is the dimensionless action variable defined as

$$
S=4 \int_{\vartheta_{1}}^{\pi / 2} \sqrt{\sigma \cos ^{2} \vartheta+\ln \sin \vartheta} d \vartheta
$$

$\vartheta_{1}$ is the smallest root of the separatrix $\sigma \cos ^{2} \vartheta+\ln \sin \vartheta$ $=0$ (trajectory on which escape may take place), and the depopulation factor is

$$
A(d)=\exp \left\{\frac{2}{\pi} \int_{0}^{\pi / 2} \ln \left[1-e^{-d /\left(4 \cos ^{2} x\right)}\right] d x\right\} .
$$

Furthermore, for a double-well potential the TST escape rate $\Gamma_{\text {TST }}$ is $^{12}$

$$
\Gamma_{\mathrm{TST}} \sim \frac{e^{-V^{\prime}(\pi / 2) / k_{B} T}}{2 \eta \sqrt{\pi} \int_{0}^{\pi / 2} e^{-V^{\prime}(\vartheta) / k_{B} T} d \vartheta}=\frac{\sigma-1 / 2}{\eta \sqrt{\pi}} e^{-\sigma} .
$$

The depopulation factor $A(d)$ as may be deduced from Eq. (17) has the following asymptotic properties:

$$
A(d) \rightarrow 1 \text { as } d \rightarrow \infty \text { and } A(d) / d \rightarrow 1 \text { as } d \rightarrow 0,
$$

where $d$ is the ratio of the energy loss per cycle of a particle moving on the escape trajectory to the thermal energy. Hence, it follows that, in the low damping $\left(\beta^{\prime} \rightarrow 0\right)$ and VHD $\left(\beta^{\prime} \rightarrow \infty\right)$ limits, Eq. (15) predicts

$$
\tau_{\mathrm{VLD}} \sim\left(\Gamma_{\mathrm{TST}} \beta^{\prime} S\right)^{-1} \sim \frac{\sqrt{\pi} \eta}{4 \beta^{\prime}(\sigma-1 / 2)^{3 / 2}} e^{\sigma}
$$

and

$$
\tau_{\mathrm{VHD}} \sim \frac{\sqrt{\pi} \eta \beta^{\prime}}{2(\sigma-1 / 2)^{3 / 2}} e^{\sigma}
$$

We remark that in the low temperature limit, $\sigma \gg 1$, Eq. (20) coincides with the estimate of the longest relaxation time,

$$
\tau_{\mathrm{VHD}} \sim \eta \beta^{\prime} \sqrt{\pi} \sigma^{-3 / 2} e^{\sigma / 2}
$$

obtained in Ref. 8 from the Smoluchowski equation governing the probability density function $W(\vartheta, t)$ of the orientations of rotators in a uniaxial potential, viz., ${ }^{8}$

$$
2 \tau_{D} \frac{\partial}{\partial t} W=\frac{1}{\sin \vartheta} \frac{\partial}{\partial \vartheta}\left[\sin \vartheta\left(\frac{\partial}{\partial \vartheta} W+\sigma \sin 2 \vartheta W\right)\right],
$$

where $\tau_{D}=\eta \beta^{\prime}$ is the Debye relaxation time for isotropic noninertial rotational diffusion. The noninertial Smoluchowski Eq. (22) follows directly from the inertial FokkerPlanck Eq. (11) by integrating over the angular momenta and proceeding to the VHD limit.

\section{ANALYTIC FORMULA FOR $\tau$ IN THE VHD LIMIT}

In principle, since it involves a weighted sum over all the eigenvalues, the accurate calculation of the correlation time $\tau$ for all barrier heights is a much more complicated 
problem than the evaluation of the smallest nonvanishing eigenvalue in the low temperature limit. In the present problem, however, an accurate method of estimating $\tau$ in the VHD limit exists. This method based on the mean first passage time was suggested by Szabo ${ }^{33}$ in the particular context of the theory of polarized fluorescent emission in uniaxial liquid crystals. Nevertheless, it may be used for all other systems with dynamics governed by single variable FokkerPlanck equations because one may then calculate in integral form the correlation time $\tau_{A}$ of a dynamical variable $A(x)$ defined as the area under the curve of the normalized autocorrelation function $C_{A}(t)=\langle A[x(0)] A[x(t)]\rangle$, viz.,

$$
\tau_{A}=\frac{1}{\left\langle A^{2}\right\rangle} \int_{x_{1}}^{x_{2}} \frac{1}{W_{\mathrm{st}}(x) D^{(2)}(x)}\left[\int_{x_{1}}^{x} A(z) W_{\mathrm{st}}(z) d z\right]^{2} d x .
$$

Here \langle\rangle designates the statistical averages over the stationary (equilibrium) distribution function $W_{\mathrm{st}}[x(0)]$ with $x(0)$ defined in the range $x_{1} \leq x(0) \leq x_{2}$ and it is assumed that $\langle A\rangle$ $=0$. Thus all that is required to evaluate $\tau$ is a knowledge of the diffusion coefficient $D^{(2)}(x)$ and $W_{\text {st }}(x)$ (see, e.g., Ref. 8, Chap. 2, Sec. 2.10, for details). The advantage of Eq. (23) is that it yields a VHD asymptote, valid for all barrier heights including very low barriers, where the Mel'nikov-Meshkov method does not apply.

Since the dynamics of the system of planar rotators in the VHD limit are governed by a single variable, we can then obtain an accurate (that is, valid for all barrier heights) VHD asymptote by applying Eq. (23) to the present problem. In the high damping limit $\left(\beta^{\prime} \gg 1\right)$, the appropriate single variable Fokker-Planck (Smoluchowski) equation for the probability density function $W(\vartheta, t)$ of the orientations of rotators is Eq. (22). The correlation time $\tau$ can then be calculated from the following equation: ${ }^{8}$

$$
\begin{aligned}
\tau & =\frac{2 \eta \beta^{\prime}}{Z\left\langle z^{2}\right\rangle} \int_{-1}^{1} \frac{e^{-\sigma z^{2}}}{1-z^{2}}\left[\int_{-1}^{z} x e^{\sigma x^{2}} d x\right]^{2} d z \\
& =\frac{3 e^{\sigma} \eta \beta^{\prime}}{\sigma^{2} M(3 / 2,5 / 2, \sigma)} \int_{0}^{1} \frac{\cosh \left[\sigma\left(1-z^{2}\right)\right]-1}{1-z^{2}} d z,
\end{aligned}
$$

where $Z=\int_{-1}^{1} e^{\sigma z^{2}} d z=2 M(1 / 2,3 / 2, \sigma)$ is the partition function, $M(3 / 2,5 / 2, \sigma)$ and $M(1 / 2,3 / 2, \sigma)$ are confluent hypergeometric (Kummer's) functions, ${ }^{23}$ and $z=\cos \vartheta$. In the low temperature limit, $\sigma \gg 1$, Eq. (24) can be approximated by Eq. (21). In the opposite high temperature limit of small barriers, $\sigma \leq 1$ (where, of course, the Mel'nikov-Meshkov universal formula is not applicable), Eq. (24) may also be used to estimate $\tau$ in the high damping limit, $\beta^{\prime} \gg 1$ (see Fig. 2).

\section{RESULTS AND DISCUSSION}

In Fig. 2, we compare the longest relaxation time $\tau_{M}$ as a function of the barrier height $\sigma$ calculated from the Mel'nikov-Meshkov Eq. (15) with numerical solutions of Eqs. (7) and (8) for the correlation time $\tau$ for low $\left(\beta^{\prime}\right.$ $=0.02,0.2)$, intermediate $\left(\beta^{\prime}=1.0\right)$, and high $\left(\beta^{\prime}=10,100\right)$ values of damping. In Fig. 3, we compare $\tau_{M}$ as a function of the damping parameter $\beta^{\prime}$ with the numerical solution for $\tau$

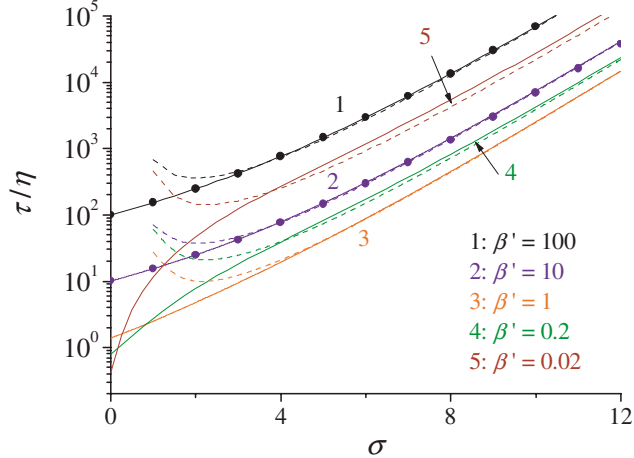

FIG. 2. (Color online) Normalized relaxation time $\tau / \eta$ vs the barrier height (inverse temperature) parameter $\sigma$ for $\beta^{\prime}=100$ (curve 1), $\beta^{\prime}=10$ (curve 2), $\beta^{\prime}=1$ (curve 3), $\beta^{\prime}=0.2$ (curve 4), and $\beta^{\prime}=0.02$ (curve 5). Solid lines: Exact matrix continued fraction solution for the correlation time, Eq. (10), dashed lines: The universal Mel'nikov-Meshkov Eq. (15); filled circles: Eq. (24) for $\beta^{\prime}=10$ and 100.

and with the VLD and VHD asymptotes, Eqs. (19) and (24), respectively. Apparently, in the high barrier limit, say $\sigma>3$, Eq. (15) provides a good approximation to the exact correlation time for all values of the friction parameter $\beta^{\prime}$ including the VHD, VLD, and the Kramers turnover regions. In spite of very good overall agreement between numerical results and the universal Eq. (15), a marked difference between numerical and analytical results exists in the VLD region at moderate barriers (this difference decreases with increasing $\sigma)$. This difference has already been noted for other systems (see, e.g., Refs. 14 and 34). In order to improve the accuracy of the universal turnover formula in this region, Mel'nikov ${ }^{35}$ suggested a systematic way of accounting for finite-barrier corrections. If such a correction is included, the accuracy of the universal formula is improved (see, e.g., Ref. 34).

The real and imaginary parts of the spectra of the dipole correlation function $\widetilde{C}(\omega)$ for various values of the barrier height $\sigma$ and the friction coefficient $\beta^{\prime}$ are shown in Figs. 4 and 5. Clearly one relaxation process dominates the lowfrequency part of the spectra and is due to the slow overbarrier relaxation of the molecules in the double-well potential.

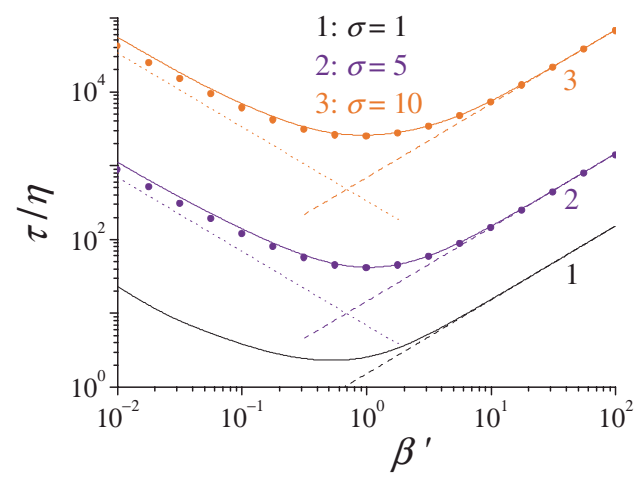

FIG. 3. (Color online) $\tau / \eta$ vs the damping parameter $\beta^{\prime}$ for barrier height parameters $\sigma=1,5$, and 10. Solid lines 1: Exact matrix continued fraction solution for the correlation time, Eq. (10); filled circles: The universal Mel'nikov-Meshkov Eq. (15); straight dashed lines: The VHD Eq. (24); dotted line 3: The VLD Eq. (19). 


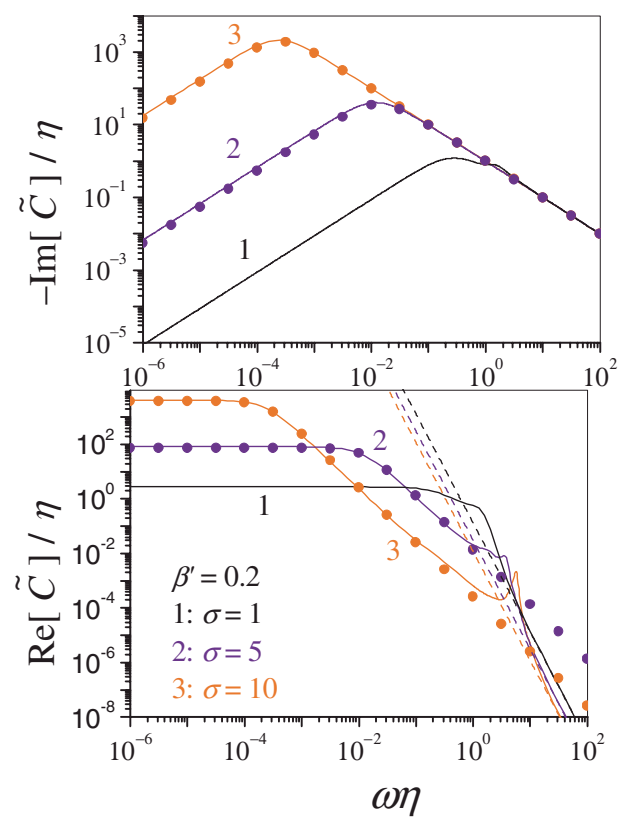

FIG. 4. (Color online) Real and imaginary parts of the spectra of the dipole correlation function $\widetilde{C}(\omega)$ for various values of the barrier height $\sigma$ $=1,5,10$, and the damping parameter $\beta^{\prime}=0.2$. Solid lines 1: Exact matrix continued fraction solution, Eq. (9); filled circles: The single Lorentzian Eq. (25); straight dashed lines: The high-frequency asymptote Eq. (27).

Clearly as may be seen from Figs. 4 and 5, the lowfrequency part of the spectrum may be approximated by a single Lorentzian,

$$
\widetilde{C}_{D}(\omega)=\frac{\tau_{M}}{1+i \omega \tau_{M}} .
$$

The characteristic frequency $\omega_{R} \approx \tau_{M}^{-1}$ and the half-width $\Delta \omega_{R} \approx \tau_{M}^{-1}$ of this low-frequency band strongly depends on $\sigma$

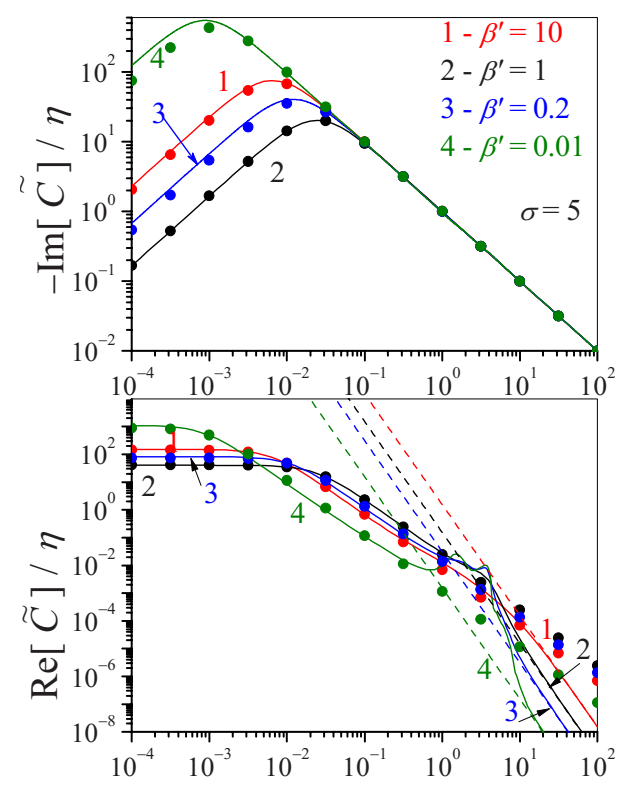

$\omega \eta$

FIG. 5. (Color online) Real and imaginary parts of the spectra of the dipole correlation function $\widetilde{C}(\omega)$ for various values of the damping parameter $\beta^{\prime}$ $=10,1,0.2,0.01$, and the barrier height $\sigma=5$. Solid lines 1: Matrix continued fraction solution, Eq. (9); filled circles: The single Lorentzian Eq. (25); straight dashed lines: The high-frequency asymptote Eq. (27).

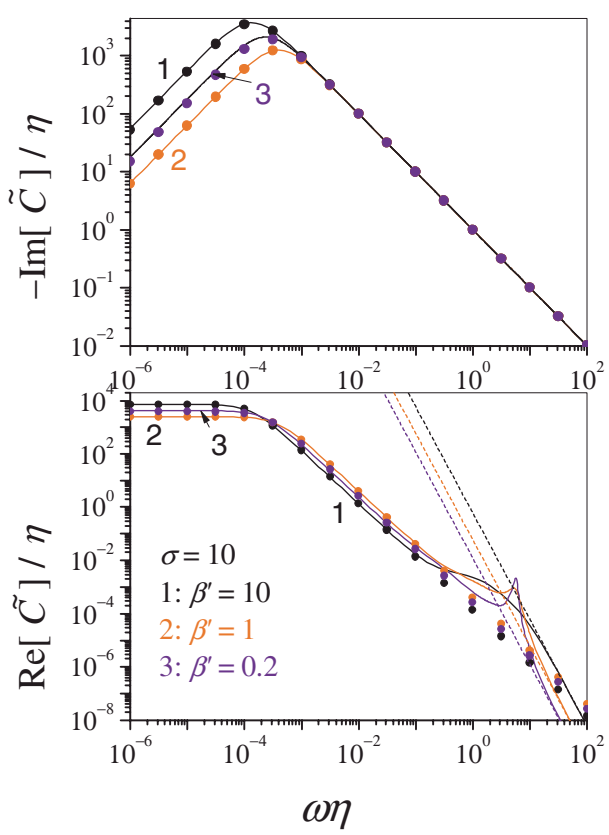

FIG. 6. (Color online) The same as in Fig. 5 for various values of $\beta^{\prime}$ $=10,1,0.2$ and $\sigma=10$.

as well as on the friction parameter $\beta^{\prime}$. Regarding the barrier height $\sigma$, the frequency $\omega_{R}$ decreases exponentially as $\sigma$ is raised. This behavior occurs because the probability of escape of a dipole from one well to another over the potential barrier exponentially decreases with increasing $\sigma$. As far as the dependence of the low-frequency part of the spectrum for high damping (small inertial effects) $\beta^{\prime}>10$ is concerned, the frequency $\omega_{R}$ decreases as $\beta^{\prime}$ increases as is apparent by inspection of curves $\operatorname{Im}[\widetilde{C}(\omega)]$ and $\operatorname{Re}[\widetilde{C}(\omega)]$ in Fig. 5. For low damping (large inertial effects) $\beta^{\prime}<0.1$ the frequency $\omega_{R}$ decreases with decreasing $\beta^{\prime}$ for given values of $\sigma$. A very high-frequency resonance band due to the fast inertial librations of the dipoles in the potential wells is also visible in Figs. 4-6. For $\sigma \gg 1$, the characteristic frequency of libration $\omega_{L}$ increases as $\sim \sqrt{\sigma / 2} \eta^{-1}$ with increasing $\sigma$. As far as the behavior as a function of $\beta^{\prime}$ is concerned, the amplitude of the high-frequency band decreases progressively with increasing $\beta^{\prime}$, as one would intuitively expect. On the other hand, for low damping (large inertial effects) $\beta^{\prime} \ll 1$, a fine structure appears in the high-frequency part of the spectra [due to resonances at high harmonic frequencies of the librations in the (anharmonic) potential]. This is the dielectric analog of the comblike structure discernible in the highfrequency ferromagnetic resonance of a superparamagnet arising from the Larmor precession in a uniaxial potential. ${ }^{8}$ Finally, it is apparent that between the low-frequency relaxation and very high-frequency resonance bands, a third band can appear in the spectra. This band is due to the highfrequency relaxation or decay modes of the dipoles in the potential wells which will always exist in the spectra even in the noninertial limit. ${ }^{8}$ Such relaxation modes are generally termed the intrawell relaxation modes. 
By utilizing general properties of Fourier transforms, we can also obtain an asymptotic expansion for $\widetilde{C}(\omega)$ in the high-frequency limit, $\omega \rightarrow \infty$, viz.,

$$
\widetilde{C}(\omega)=\int_{0}^{\infty} C(t) e^{i \omega t} d t \sim \frac{i}{\omega}-\frac{i \ddot{C}(0)}{\omega^{3}}+\frac{\dddot{C}(0)}{\omega^{4}} \cdots
$$

so that $\operatorname{Im}[\widetilde{C}(\omega)] \sim \omega^{-1}+\cdots$ and

$$
\operatorname{Re}[\widetilde{C}(\omega)] \sim \dddot{C}(0) \omega^{-4}+\cdots
$$

[here we have noted that $C(0)=1$ and $\dot{C}(0)=\dot{c}_{0}^{1,0}(0) / c_{0}^{1,0}(0)$ $=0]$. The high-frequency asymptote Eq. (27) is also shown in Figs. 4 and 5 for comparison. We remark that for $\sigma=0$, the calculated spectrum of the normalized correlation function $\widetilde{C}(\omega)$ coincides with that obtained by Sack $^{36}$ for inertial rotational diffusion of free linear molecules and is given by the infinite continued fraction,

$$
\tilde{C}(\omega)=\frac{\eta}{i \omega \eta+\frac{1}{\beta^{\prime}+i \omega \eta+\frac{1}{2 \beta^{\prime}+i \omega \eta+\frac{2}{3 \beta^{\prime}+i \omega \eta+\frac{2}{4 \beta^{\prime}+i \omega \eta+\cdots}}}}} .
$$

Thus we have demonstrated how the matrix continued fraction approach for the solution of nonlinear Langevin equations $^{27}$ may be successfully applied to the rotational Brownian motion of a linear molecule in a uniaxial potential, Eq. (1), for wide ranges of the barrier height parameter $\sigma$ and the damping parameter $\beta^{\prime}$. We have shown that in the lowtemperature limit, the Mel'nikov-Meshkov formula, Eq. (15), for the longest relaxation time yields satisfactory agreement with the numerical results for all values of damping. Moreover, the Mel'nikov-Meshkov Eq. (15) allows one to estimate accurately the damping dependence of the lowfrequency parts of the spectra of the equilibrium dipole correlation function $C(t)$. We have given exact, as well as simple, approximate analytic formulas for the correlation time $\tau$ and the spectra of the dipole correlation function $\widetilde{C}(\omega)$. The model may be used to interpret broadband $(0$ $\mathrm{THz}$ ) dielectric spectra of nematic liquid crystals. These are related to the microscopic parameters (damping $\beta$ and the barrier height $\sigma$ ), which are closely associated with the molecular dynamics. The noninertial rotational diffusion model is only applicable to the low-frequency range $(\omega \tau<1$, where $\tau$ is the dipole moment relaxation time). The model of the inertial rotational diffusion in a mean field potential may, however, also be used in the high-frequency range (up to -5 $\mathrm{THz}$ ). At low frequencies the model describes the relaxation (Debye) spectrum just as the noninertial rotational diffusion model. In particular, the results are in agreement with the available experimental data (e.g., Ref. 19) where the longitudinal relaxation time $\tau$ increases in nematic liquid crystals in comparison to the relaxation time in the isotropic phase. In the far infrared range, the model predicts the characteristic frequency of the librational (Poley) absorption band. ${ }^{8}$ However, for a quantitative comparison with experimental data one will also need to evaluate the transverse component of the complex susceptibility which may again be accomplished using the Langevin equation method.

\section{ACKNOWLEDGMENTS}

We thank the Trinity College Dublin Trust for a Grant in support of this work.

\section{APPENDIX: MATRIX CONTINUED FRACTION SOLUTION OF EQS. (7) and (8)}

The hierarchy of the differential-recurrence Eqs. (7) and (8) for $c_{n}^{l, m}(t)$ can be transformed into the matrix three-term differential-recurrence equation,

$$
\eta \frac{d}{d t} \mathbf{C}_{n}(t)=\mathbf{Q}_{n}^{-} \mathbf{C}_{n-1}(t)+\mathbf{Q}_{n} \mathbf{C}_{n}(t)+\mathbf{Q}_{n}^{+} \mathbf{C}_{n+1}(t) .
$$

Here we have introduced the vectors

$$
\mathbf{C}_{n}=\left(\begin{array}{c}
\mathbf{c}_{n-1}^{1} \\
\mathbf{c}_{n-1}^{2} \\
\vdots \\
\mathbf{c}_{n-1}^{l_{\max }}
\end{array}\right)_{\left(l_{\max }+1\right) l_{\max }} \quad, \ldots \mathbf{c}_{n}^{l}=\left(\begin{array}{c}
\mathbf{c}_{n}^{2 l-1,0} \\
\mathbf{c}_{n}^{2 l-1,1} \\
\vdots \\
c_{n}^{2 l-1,2 l-1}
\end{array}\right)_{2 l}
$$

with $\mathbf{C}_{0}=\mathbf{0}$ and the matrices 


$$
\begin{aligned}
& \mathbf{Q}_{n}=\left(\begin{array}{ccccc}
\mathbf{q}_{n-1,1} & \mathbf{p}_{n-1,1}^{+} & \mathbf{0} & \ldots & \mathbf{0} \\
\mathbf{p}_{n-1,2}^{-} & \mathbf{q}_{n-1,2} & \mathbf{p}_{n-1,2}^{+} & \ldots & \mathbf{0} \\
\mathbf{0} & \mathbf{p}_{n-1,3}^{-} & \ddots & \ddots & \vdots \\
\vdots & \vdots & \ddots & \mathbf{q}_{n-1, l_{\max }-1} & \mathbf{p}_{n-1, l_{\max }-1}^{+} \\
\mathbf{0} & \mathbf{0} & \ldots & \mathbf{p}_{n-1, l_{\max }}^{-} & \mathbf{q}_{n-1, l_{\max }}
\end{array}\right), \\
& \mathbf{Q}_{n}^{+}=\left(\begin{array}{ccccc}
\mathbf{q}_{n-1,1}^{+} & \mathbf{0} & \mathbf{0} & \ldots & \mathbf{0} \\
\mathbf{0} & \mathbf{q}_{n-1,2}^{+} & \mathbf{0} & \ldots & \mathbf{0} \\
\mathbf{0} & \mathbf{0} & \ddots & \ddots & \vdots \\
\vdots & \vdots & \ddots & \mathbf{q}_{n-1, l_{\max }^{+}}^{+} & \mathbf{0} \\
\mathbf{0} & \mathbf{0} & \ldots & \mathbf{0} & \mathbf{q}_{n-1, l_{\max }}^{+}
\end{array}\right) \\
& \mathbf{Q}_{n}^{-}=\left(\begin{array}{ccccc}
\mathbf{q}_{n-1,1}^{-} & \mathbf{r}_{n-1,1}^{+} & \mathbf{0} & \cdots & \mathbf{0} \\
\mathbf{r}_{n-1,2}^{-} & \mathbf{q}_{n-1,2}^{-} & \mathbf{r}_{n-1,2}^{+} & \cdots & \mathbf{0} \\
\mathbf{0} & \mathbf{r}_{n-1,3}^{-} & \ddots & \ddots & \vdots \\
\vdots & \vdots & \ddots & \mathbf{q}_{n-1, l_{\max }-1}^{-} & \mathbf{r}_{n-1, l_{\max }^{+}}^{+} \\
\mathbf{0} & \mathbf{0} & \cdots & \mathbf{r}_{n-1, l_{\max }}^{-} & \mathbf{q}_{n-1, l_{\max }}^{-}
\end{array}\right),
\end{aligned}
$$

where $\mathbf{q}_{n, l}, \mathbf{q}_{n, l}^{ \pm}, \mathbf{r}_{n, l}^{ \pm}$, and $\mathbf{p}_{n, l}^{ \pm}$,

$$
\begin{aligned}
& {\left[\mathbf{q}_{n, l}\right]_{i, j=1}^{2 l, 2 l}= \begin{cases}-\beta^{\prime} 2 n \delta_{i, j}+\frac{1+\delta_{i, 1}}{4} \delta_{i, j-1}-\frac{1}{4}\left(n+1-\frac{i-1}{2}\right)(2 l+i-2)(2 l-i+1) \delta_{i, j+1} & (i: \text { odd }) \\
-\beta^{\prime}(2 n+1) \delta_{i, j}+\left[1+\frac{2 \sigma(2 i-1)}{(4 l-3)(4 l+1)}\right] \delta_{i, j-1} & \\
-\left(n+\frac{i}{2}\right)(2 l+i-2)(2 l-i+1)\left[1-\frac{2 \sigma(2 i-3)}{(4 l-3)(4 l+1)}\right] \delta_{i, j+1} & \text { (i:even) }\end{cases} } \\
& {\left[\mathbf{q}_{n, l}^{+}\right]_{i, j=1}^{2 l, 2 l}=\frac{1}{4}\left(\delta_{i, j-1}-\left(n-\frac{i}{2}+2\right)(2 l+i-2)(2 l-i+1) \delta_{i, j+1}\right) \quad(i: \text { even }),} \\
& {\left[\mathbf{q}_{n, l}^{-}\right]_{i, j=1}^{2 l, 2 l}=\left(1+\delta_{i, 1}\right)\left[1+\frac{2 \sigma(2 i-1)}{(4 l-3)(4 l+1)}\right] \delta_{i, j-1}-\left(n+\frac{i-1}{2}\right)(2 l+i-2)(2 l-i+1)\left[1-\frac{2 \sigma(2 i-3)}{(4 l-3)(4 l+1)}\right] \delta_{i, j+1}(i: \text { odd })} \\
& {\left[\mathbf{p}_{n, l}^{+}\right]_{i, j=1}^{2 l, 2(l+1)}=\frac{2 \sigma(2 l-i+1)}{(4 l-1)(4 l+1)}\left[\delta_{i, j-1}-\left(n+\frac{i}{2}\right)(2 l-i+2)(2 l-i+3) \delta_{i, j+1}\right] \quad(i: \text { even }),} \\
& {\left[\mathbf{p}_{n, l}^{-}\right]_{i, j=1}^{2 l, 2(l-1)}=-\frac{2 \sigma(2 l+i-2)}{(4 l-3)(4 l-1)}\left[\delta_{i, j-1}-\left(n+\frac{i}{2}\right)(2 l+i-3)(2 l+i-4) \delta_{i, j+1}\right] \quad(i: \text { even }),} \\
& {\left[\mathbf{r}_{n, l}^{+}\right]_{i, j=1}^{2 l, 2(l+1)}=\frac{2 \sigma(2 l-i+1)}{(4 l-1)(4 l+1)}\left[\left(1+\delta_{i, 1}\right) \delta_{i, j-1}-\left(n+\frac{i-1}{2}\right)(2 l-i+2)(2 l-i+3) \delta_{i, j+1}\right] \quad(i: \text { odd }),} \\
& {\left[\mathbf{r}_{n, l}^{-}\right]_{i, j=1}^{2 l, 2(l-1)}=-\frac{2 \sigma(2 l+i-2)}{(4 l-3)(4 l-1)}\left[\left(1+\delta_{i, 1}\right) \delta_{i, j-1}-\left(n+\frac{i-1}{2}\right)(2 l+i-3)(2 l+i-4) \delta_{i, j+1}\right] \quad(i: \text { odd }) .}
\end{aligned}
$$


All other matrix elements are zero. In calculations, the value of $l_{\max }$ was determined in such way that a further increase in $l_{\max }$ did not change the result $\left(l_{\max }\right.$ depends on the dimensionless barrier $\sigma$ and damping $\beta^{\prime}$ parameters and must be chosen taking into account the desired degree of accuracy of the calculation)

The exact solution of Eq. (A1) for the Laplace transform $\widetilde{\mathbf{C}}_{1}(s)=\int_{0}^{\infty} \mathbf{C}_{1}(t) e^{-s t} d t$ is then given in terms of matrix continued fractions as 8,37

$$
\widetilde{\mathbf{C}}_{1}(s)=\eta \boldsymbol{\Delta}_{1}(s) \mathbf{C}_{1}(0),
$$

where $\Delta_{1}(s)$ is the infinite matrix continued fraction defined as

$$
\boldsymbol{\Delta}_{1}(s)=\frac{\mathbf{I}_{1}}{\eta s \mathbf{I}_{1}-\mathbf{Q}_{1}-\mathbf{Q}_{1}^{+} \frac{\mathbf{I}_{2}}{\eta s \mathbf{I}_{2}-\mathbf{Q}_{2}-\mathbf{Q}_{2}^{+} \frac{\mathbf{I}_{3}}{\eta s \mathbf{I}_{3}-\mathbf{Q}_{3}-\ddots} \mathbf{Q}_{3}^{-}} \mathbf{Q}_{2}^{-}}
$$

(here the fraction lines denote matrix inversions and $\mathbf{I}_{n}$ are the identity matrices). The initial value vector $\mathbf{C}_{1}(0)$ in $\mathrm{Eq}$. (A2) is given by

$$
\mathbf{C}_{1}(0)=\left(\begin{array}{c}
\mathbf{c}_{0}^{1}(0) \\
\mathbf{c}_{0}^{2}(0) \\
\vdots \\
\mathbf{c}_{0}^{l_{\max }(0)}
\end{array}\right) \quad, \ldots \mathbf{c}_{0}^{l}(0)=\left(\begin{array}{c}
c_{0}^{2 l-1,0} \\
0 \\
\vdots \\
0
\end{array}\right)_{2 l}{ }_{\left.l_{\max }+1\right) l_{\max }},
$$

where

$$
c_{0}^{2 n-1,0}=\left\langle P_{1} P_{2 n-1}\right\rangle=\frac{2 n}{4 n-1}\left\langle P_{2 n}\right\rangle+\frac{2 n-1}{4 n-1}\left\langle P_{2 n-2}\right\rangle
$$

and $\left\langle P_{2 n}\right\rangle$ are the equilibrium averages of the Legendre polynomials. Here we have noted that $c_{n}^{l, m}(0)=0$ for $n \neq 0$ and $m \neq 0$. The equilibrium averages $\left\langle P_{2 n}\right\rangle$ satisfy the following recurrence equation: ${ }^{8}$

$$
\begin{aligned}
(1- & \left.\frac{2 \sigma}{(4 n-1)(4 n+3)}\right)\left\langle P_{2 n}\right\rangle-\frac{2 \sigma(2 n-1)}{(4 n-1)(4 n+1)}\left\langle P_{2 n-2}\right\rangle \\
& +\frac{2 \sigma(2 n+2)}{(4 n+3)(4 n+1)}\left\langle P_{2 n+2}\right\rangle=0
\end{aligned}
$$

and can be calculated as ${ }^{8}\left\langle P_{2 n}\right\rangle=\prod_{j=1}^{n} S_{2 j}$, where the scalar continued fraction $S_{n}$ is defined by the recurrence equation ${ }^{8}$

$$
\begin{aligned}
S_{n}= & \frac{2 \sigma(n-1)}{4 n^{2}-1}\left[1-\frac{2 \sigma}{(2 n-1)(2 n+3)}\right. \\
& \left.+\frac{2 \sigma(n+2)}{(2 n+1)(2 n+3)} S_{n+2}\right]^{-1} .
\end{aligned}
$$

We remark the initial value $\dddot{C}(0)=\dddot{c}_{0}^{1,0}(0) / c_{0}^{1,0}(0)$ appearing in Eq. (27) can be found from vectors $\ddot{\mathbf{C}}_{1}(0)$ and $\mathbf{C}_{1}(0)$. Noting that $\mathbf{C}_{n}(0)=\mathbf{0}$ for $n>1$, one can show that

$\eta^{3} \dddot{\mathbf{C}}_{1}(0)=\left[\mathbf{Q}_{1}\left(\mathbf{Q}_{1} \mathbf{Q}_{1}+\mathbf{Q}_{1}^{+} \mathbf{Q}_{2}^{-}\right)+\mathbf{Q}_{1}^{+}\left(\mathbf{Q}_{2}^{-} \mathbf{Q}_{1}+\mathbf{Q}_{2} \mathbf{Q}_{2}^{-}\right)\right] \mathbf{C}_{1}(0)$.

${ }^{1}$ G. Meier and A. Saupe, Mol. Cryst. 1, 515 (1966).

${ }^{2}$ A. J. Martin, G. Meier, and A. Saupe, Symp. Faraday Soc. 5, 119 (1971). ${ }^{3}$ P. L. Nordio and P. Busolin, J. Chem. Phys. 55, 5485 (1971).

${ }^{4}$ P. L. Nordio, G. Rigatti, and U. Segre, J. Chem. Phys. 56, 2117 (1972); Mol. Phys. 25, 129 (1973).

${ }^{5}$ B. A. Storonkin, Kristallogr. 30, 841 (1985); [Sov. Phys. Crystallogr. 30, 489 (1985)].

${ }^{6}$ W. T. Coffey and Yu. P. Kalmykov, Adv. Chem. Phys. 113, 487 (2000).

${ }^{7}$ B. U. Felderhof, Physica A 323, 88 (2003).

${ }^{8}$ W. T. Coffey, Yu. P. Kalmykov, and J. T. Waldron, The Langevin Equation, 2nd ed. (World Scientific, Singapore, 2004).

${ }^{9}$ M. L. Huertas, V. Cruz, J. J. Lopez Cascales, A. U. Acuha, and J. Garcia de la Torre, Biophys. J. 71, 1428 (1996).

${ }^{10}$ M. X. Fernandes, M. L. Huertas, M. A. R. B. Castanho, and J. Garcia de la Torre, Biophys. J. 79, 41 (1999).

${ }^{11}$ B. Lassier and C. Brot, Discuss. Faraday Soc. 48, 39 (1969).

${ }^{12}$ R. W. Pastor and A. Szabo, J. Chem. Phys. 97, 5098 (1992).

${ }^{13}$ F. Marchesoni and J. K. Vij, Z. Phys. B: Condens. Matter 58, 187 (1985); F. Marchesoni, Phys. Rev. B 32, 1827 (1985)

${ }^{14}$ W. T. Coffey, Yu. P. Kalmykov, and S. V. Titov, J. Chem. Phys. 120, 9199 (2004); Yu. P. Kalmykov, S. V. Titov, and W. T. Coffey, ibid. 123, 094503 (2005).

${ }^{15}$ W. F. Brown, Jr., Phys. Rev. 130, 1677 (1963); IEEE Trans. Magn. 15, 1196 (1979)

${ }^{16}$ W. T. Coffey, Yu. P. Kalmykov, and P. J. Cregg, Adv. Chem. Phys. 83, 263 (1993); W. T. Coffey, D. S. F. Crothers, Yu. P. Kalmykov, E. S. Massawe, and J. T. Waldron, Phys. Rev. E 49, 1869 (1994).

${ }^{17}$ H. Watanabe and A. Morita, Adv. Chem. Phys. 56, 255 (1984).

${ }^{18}$ J. R. McConnell, Rotational Brownian Motion and Dielectric Theory (Academic, New York, 1980).

${ }^{19}$ M. W. Evans, G. J. Evans, W. T. Coffey, and P. Grigolini, Molecular Dynamics and Theory of Broadband Spectroscopy (Wiley, New York, 1982).

${ }^{20}$ A. I. Burshtein and S. I. Temkin, Spectroscopy of Molecular Rotation in Gases and Liquids (Cambridge University Press, Cambridge, 1994).

${ }^{21}$ W. T. Coffey, J. Chem. Phys. 93, 724 (1990); 95, 2026 (1991).

${ }^{22}$ S. V. Titov, Yu. P. Kalmykov, and W. T. Coffey, J. Chem. Phys. 129, 144505 (2008).

${ }^{23}$ Handbook of Mathematical Functions, edited by M. Abramowitz and I. Stegun (Dover, New York, 1964).

${ }^{24}$ H. A. Kramers, Physica (Utrecht) 7, 284 (1940).

${ }^{25}$ P. Hänggi, P. Talkner, and M. Borkovec, Rev. Mod. Phys. 62, 251 (1990).

${ }^{26}$ W. T. Coffey, D. A. Garanin, and D. J. McCarthy, Adv. Chem. Phys. 117, 528 (2001).

${ }^{27}$ E. Pollak and P. Talkner, Chaos 15, 026116 (2005).

${ }^{28}$ V. I. Mel'nikov, Physica A 130, 606 (1985); Phys. Rep. 209, 1 (1991)

${ }^{29}$ V. I. Mel'nikov and S. V. Meshkov, J. Chem. Phys. 85, 1018 (1986).

${ }^{30}$ H. Grabert, Phys. Rev. Lett. 61, 1683 (1988).

${ }^{31}$ E. Pollak, H. Grabert, and P. Hänggi, J. Chem. Phys. 91, 4073 (1989).

${ }^{32}$ I. Rips and E. Pollak, Phys. Rev. A 41, 5366 (1990).

${ }^{33}$ A. Szabo, J. Chem. Phys. 72, 4620 (1980).

${ }^{34}$ R. Ferrando, R. Spadacini, G. E. Tommei, and V. I. Mel'nikov, Phys. Rev. E 51, R1645 (1995).

${ }^{35}$ V. I. Mel'nikov, Phys. Rev. E 48, 3271 (1993); 50, 627 (1994).

${ }^{36}$ R. A. Sack, Proc. Phys. Soc. London, Sect. B 70, 414 (1957).

${ }^{37}$ H. Risken, The Fokker-Planck Equation, 2nd ed. (Springer, Berlin, 1989). 